Objectives The aim of this study was to investigate if mental health status in childhood determined future labour market participation, and to identify if effects varied across gender and social strata.

Method Of a cohort of 3681 born in 1989 in the county of Ringkjoebing, Denmark, 3058 (83\%) completed a questionnaire in 2004. They were followed in a register on social benefits for 12 months in 2010-2011. Logistic regression was used to investigate associations between mental health in childhood measured with The Centre for Epidemiological Studies Depression Scale for Children (CES-DC) and future labour market participation, taking into account effects of socio-economic position, school performance, educational plans and vocational expectations.

Results A total of $17.1 \%$ (19.9\% males, $14.4 \%$ females) received social benefits for at least 4 weeks during follow-up. Girls scored significantly lower on mental health than did boys. Labour market participation in early adulthood decreased with poor mental health in childhood, but only for boys: Boys with a baseline CES-DC score in the lowest quartile had a $70 \%$ excess risk of low labour market participation after 7 years of follow-up. The association persisted when taking into account socio-economic position, but became borderline significant when adjusting for school performance, educational plans and vocational expectations. The negative effect was even across social strata.

Conclusions Despite girls scoring significantly lower on mental health than do boys, the effects on future labour market participation was only present among boys. The effect of poor mental health on future labour market participation did not vary across social strata.

\section{SURVEILLANCE OF MORTALITY BY SUICIDE AMONG FRENCH FARMERS}

Claire Bossard, Gaëlle Santin, Irina Guseva Canu, Christine Cohidon, Ellen Imbernon. Institut de Veille Sanitaire, Saint-Maurice, France

\subsection{6/oemed-2014-102362.56}

Objectives An excessive risk of suicide among agriculture workers has been observed in several studies in France and abroad. Accordingly, French Institute for Public Health Surveillance and Social Insurance in agriculture sector launched collaboration with aim at producing indicators of suicide mortality among agriculture workers population on a regular basis.

Method The study population included all active farmers and their collaborating spouses. The study covered tree consecutive years: 2007 to 2009 . Mortality data by cause from death registrations were used to calculate standardised mortality ratios (SMRs) for both men and women.

Results The annual study population was around 500000 subjects in average, including $68 \%$ of men. During the 3 -year study period, 2769 men and 997 women were deceased. From these deaths, 417 deaths were due to suicide among men and 68 deaths among women. Suicides represented the third most important cause of death. The comparison of mortality among male study population with that of French national male population revealed a $28 \%$-excess in mortality by suicide in 2008 and $22 \%$-excess in 2009. This excess was particularly high among the 45-64 year age category and in cattle breeding-dairy and meat -sectors. These sectors presented the highest over mortality due to suicide in 2008 and 2009.

Conclusions The first results of this study confirm the necessity to continue the surveillance in this population. Especially, further analysis could provide more information to document risk factors of this excess.

\section{9}

MODELLING OF OCCUPATIONAL EXPOSURE TO HEXAVALENT CHROMIUM

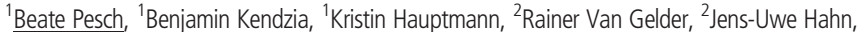
${ }^{1}$ Wolfgang Zschiesche, ${ }^{1}$ Thomas Behrens, ${ }^{1}$ Thomas Brüning. 'Institute for Prevention and Occupational Medicine of the German Social Accident Insurance - Institute of Ruhr University Bochum (IPA), Bochum, Germany; ${ }^{2}$ Institute for Occupational Safety and Health of the German Social Accident Insurance (IFA), Sankt Augustin, Germany

\subsection{6/oemed-2014-102362.57}

Objectives To assess level and trend of exposure to hexavalent chromium $(\mathrm{Cr}(\mathrm{VI}))$ in chromium-exposed occupations for the estimation of lung cancer risks in community-based studies.

Method This analysis was based on 3666 personal measurements and auxiliary data compiled in the German MEGA database from 1988-2009. Cr(VI) was determined spectrophotometrically at $540 \mathrm{~nm}$ after reaction with diphenylcarbazide. We assigned jobs tasks with known $\mathrm{Cr}(\mathrm{VI})$ exposure using coded and textual information about the workplaces. Measurements below the limit of quantification (LOQ) were multiply imputed according to their distribution above LOQ. Statistical modelling was performed to the log-transformed $\mathrm{Cr}(\mathrm{VI})$ concentrations to adjust for duration and reason of sampling.

Results $\mathrm{Cr}(\mathrm{VI})$ exposure could be assessed for eight out of 30 jobs tasks with known $\mathrm{Cr}(\mathrm{VI})$ exposure. The majority of measurements $(53 \%)$ were collected in welders $(\mathrm{N}=1930)$, which we further detailed by welding technique. Spray painting and the welding of stainless steel with shielded metal welding were associated with adjusted geometric means above $5 \mu \mathrm{g} / \mathrm{m}^{3}$, the permissible exposure level of the U.S. Occupational Safety and Health Administration. We could not detect an overall time trend in the $\mathrm{Cr}(\mathrm{VI})$ concentrations.

Conclusions Exposure to $\mathrm{Cr}(\mathrm{VI})$ varied by occupation and job task, particularly between welding techniques, but less across calendar time. Supplemental occupational questionnaires should be administered in community-based studies when estimating the lung cancer risk of $\mathrm{Cr}(\mathrm{VI})$.

\section{RESPIRATORY EFFECTS OF AN EXPOSURE TO WHEAT DUST AMONG GRAIN WORKERS AND FARMERS: A LONGITUDINAL STUDY}

1,2 Pascal Wild, ${ }^{2}$ Victor Dorribo, ${ }^{2}$ Jacques Pralong, ${ }^{3}$ Gabriel Reboux, ${ }^{2}$ Anne Oppliger, ${ }^{2}$ Brigitta Danuser, ${ }^{2}$ Peggy Krief, ${ }^{2}$ Hélène Niculita-Hirzel. ${ }^{1}$ INRS, Vandoeuvre, France; ${ }^{2}$ IST, Lausanne, Switzerland; ${ }^{3}$ University Hospital, Besançon, France

\subsection{6/oemed-2014-102362.58}

Objectives Agriculture is considered one of the occupations most at risk of acute or chronic respiratory problems. The aim of our study was to determine from which level of exposure to organic dust the respiratory function is chronically affected in workers involved in wheat grain or straw manipulation and to test if some of these working populations can recover their respiratory function after an exposure decrease.

Method 87 workers exposed to wheat dust: farmers, harvesters, silo workers and livestock farmers and 62 non exposed workers, were included into a longitudinal study comprising two visits at a six months interval with lung function measurements and symptom questionnaires. Cumulative and mean exposure to wheat dust were generated from detailed work history of each worker and a task-exposure matrix based on task-specific exposure measurements. Immunoglobulins (IgG and IgE) specific of the most frequent microorganisms in wheat dust have been determined. 